\title{
Regional consumption of antibiotics: a demand system approach
}

\author{
M. Filippini * $\quad$ G. Masiero ${ }^{\dagger} \quad$ K. Moschetti ${ }^{\ddagger}$ \\ Published in Economic Modelling (2009), 26, 6: 1389-1397, \\ http://www.sciencedirect.com/science/journal/02649993
}

\begin{abstract}
Through this paper, we have attempted to model the demand for different classes of antibiotics used for respiratory infections in outpatient care in Switzerland using a spatial version of the linear approximate Almost Ideal Demand System (AIDS) model. This model takes spatial dependency into account by means of spatial lags of antibiotic budget shares. We control for the health status of patients and the potential harmful effects of antibiotic use in terms of bacterial resistance. Elasticities to socioeconomic determinants of consumption and own- and cross-price elasticities between different groups of antibiotic have salso been computed in this paper. Significant cross-price elasticities are found between newer or more expensive generations and older or less expensive generations of antibiotics.
\end{abstract}

JEL classification: I0; C3; C43

Keywords: Antibiotic use; Demand equations; Demand elasticities; Almost Ideal Model; Bacterial resistance.

\footnotetext{
*Department of Economics, University of Lugano; ETH, Zurich, Switzerland.

${ }^{\dagger}$ Department of Economics and Technology Management, University of Bergamo, Italy; Department of Economics, University of Lugano, Switzerland. Corresponding author. Tel.: +41 058 666 4731; fax: +41058666 4733. E-mail address: giuliano.masiero@lu.unisi.ch. Support from the Swiss National Science Foundation is acknowledged. We are grateful to Dr. Enos Bernasconi and Dr. Marco Bissig from the Cantonal Hospital of Lugano for their invaluable advice on the selection of antibiotic categories. Any mistake is clearly our fault.

${ }^{\ddagger}$ Department of Economics, University of Lugano; IEMS, University of Lausanne, Switzerland.
} 


\section{Introduction}

Antibiotics differ from other pharmaceutical products in several ways (Ellison and Hellerstein, 1999). They are generally prescribed by doctors because of their pharmacological characteristics. Consequently, the patients' preferences for specific therapeutic components may be of little relevance. At the same time, aspects such as the price may play an important role if the consumer was to bear a substantial share of the cost of drugs directly.

In primary care, doctors may face a first trade-off between prescribing and delaying an antibiotic therapy because of uncertainty of the nature of a patient's infection (viral or bacterial). Although antibiotic therapy is not effective against common viral infections, expected cost savings (in terms of time and troubles) in the case of bacterial infections may overcome the expected social loss from bacterial resistance. From the economic point of view, bacterial resistance accounts for relevant external effects. First, several antibiotic therapies might be prescribed before finding the effective one, which leads to income loss or possibly premature deaths. Second, the reduced effectiveness of drugs increases social costs to produce new generations of pharmaceutical components.

A second trade-off is observed between prescribing broad spectrum and narrow spectrum antibiotics. Although the intensive use of broad spectrum components, which are generally more expensive, reduces uncertainty in the outcome of the treatment, this may generate higher levels of bacterial resistance. It has been argued that the persistent use of one type of antibiotics may be sub-optimal for the society. Literature suggests that the negative externality of resistance can be reduced by changing the type of antibiotic used (Ellison and Hellerstein 1999; Laxminarayan and Weitzman, 2002; Rowthorn and Brown, 2003), since the same type of bacteria may be susceptible to more than one antibiotic component. ${ }^{1}$

\footnotetext{
${ }^{1}$ Increasing rates of Streptococcus pneumoniae strains resistant to penicillin has led to the use of macrolides. However, the clinical effectiveness of the latter category has also begun to decrease (Alvarez-Elcoros and Enzler, 1999). The analysis by Ednie et al. (1996) suggests that in areas where penicillin-resistant strains are common, the empirical antibiotherapy using macrolides should
} 
Regional heterogeneity in the mix of antibiotics used has been recently observed within the countries (Filippini et al., 2006; Kern et al., 2006). This may indicate that some antibiotic components are preferred by local physicians and patients, which raises the issue of optimal use at the local level. The rise of resistant organisms in a particular region can force physicians to face constraints of effectiveness when substituting existing types of antibiotics with newer and more effective ones. ${ }^{2}$ Furthermore, there are other factors that influence the mix of antibiotics such as differences in attitudes towards the same type of antibiotic, patients' characteristics, antibiotic price and economic incentives.

This study focuses on factors affecting the local mix of main antibiotics categories in Switzerland, in particular the effect of prices. The demand for specific antibiotic groups has been investigated in studies by Ellison et al. (1997) and Chaudhuri et al. (2003). The focus is on the structure of the demand for two segments of the market: cephalosporins and quinolones. Demand is modelled in two stages. Firstly a substance is singled out from among a set of substances; then, a brand/generic version of the product is chosen. This approach is suitable for the analysis of specific categories of antibiotics since the substances constitute close therapeutic substitutes and may be similar in terms of the impact of bacterial resistance. The model relies on the hypothesis that physician and patient decisions within a given antibiotic category do not depend on the availability of alternative categories but are based on the specific names of substances. We argue, however, that this scenario may not reflect the patient's and doctor's view correctly. Indeed, doctors and patients tend to be concerned with the effectiveness of broad categories of antibiotics, each including a set of active ingredients with similar characteristics and, for this reason, likely to be differently affected by bacterial resistance. Individuals may then choose from among a limited set of antibiotic types clustered according to common practice and shared beliefs regarding their effectiveness.

then be changed.

${ }^{2}$ The resistance-induced antibiotic substitution has been recently addressed by Howard (2004) among others. 
This paper intends to investigate the structure of the demand for antibiotics used for respiratory infections by modelling the decision process of rational patients. ${ }^{3} \mathrm{We}$ are interested in the price and income sensibility of different antibiotic categories. Understanding the determinants of antibiotic treatment practices is necessary to reduce antibiotic misuse and the cost of bacterial resistance. Our findings may drive more effective policies to improve the efficient use of the antibiotics in the community. It has been assessed that the expected benefits of policy interventions aimed at reducing the misuse of antibiotics in Switzerland is around $€ 6.8 \mathrm{M}$ per year (Filippini et al., 2009). Appropriate incentives affecting antibiotic consumption and, consequently, levels of bacterial resistance can be designed by policy makers by looking at the price and income sensibility of different antibiotic groups in our model. Since the large use of broad spectrum antibiotics and/or the persistent use of one type of antibiotics may exacerbate the threat of bacterial resistance, information on price sensibility may be useful. For instance, when implementing policies based upon a tax/subsidy mechanism in addition to information campaigns targeted at consumers and health care providers.

Similar to Ellison et al. (1997) and Chaudhuri et al. (2003), we model antibiotic demand as a multistage budgeting problem but with a wider set of substances, those that can be prescribed for common respiratory infections in outpatient care. Outpatient antibiotics are then aggregated in four groups according to what are plausible alternatives in the treatment of respiratory infections (classic penicillins, penicillins amoxi/clav plus 1st and 2nd generations of cephalosporins, 3rd generation of cephalosporins and quinolones, macrolides). ${ }^{4}$

We analyse the allocation of antibiotic expenditure across antibiotic groups us-

\footnotetext{
${ }^{3}$ We are aware that patients take decisions about antibiotic use under doctor's advice. However, as suggested by several studies, e.g. Cockburn and Pit (1997), physicians' decisions are influenced by patient's preferences and we can plausibly assume that the final decision about consumption is made by the patient.

${ }^{4}$ Most of the antibiotics used in outpatient care are for the treatment of respiratory infections (Blasi et al., 2006; Gonzalves et al., 2001). We focus on this category and exclude antibiotics that are used for other types of infections. Hence, our categories include all plausible therapeutic substitutes.
} 
ing the Almost Ideal Demand System (AIDS) specification proposed by Deaton and Muellbauer (1980). The AIDS model is commonly used to estimate price and income elasticities of the demand for goods when expenditure share data are available. ${ }^{5}$ We compute own- and cross-price elasticities between old and new generations of antibiotics and between antibiotics that are differently affected by bacterial resistance using data from small geographic areas in Switzerland. We also estimate conditional expenditure elasticities and marginal expenditure shares for each group of antibiotics. The AIDS model is estimated using a relatively new econometric approach that also considers the problem of spatial autocorrelation.

The structure of the article is as follows. Section 2 sketches the application of the linear approximate AIDS model to the demand of antibiotics and discusses some testable hypothesis. Section 3 presents the data and summarizes the variables used in the model. Section 4 gives some analyses and results. And section 5 concludes the paper.

\section{The model}

To sketch the demand for antibiotics in outpatient care we build on the AIDS model and extend the approach to include spatial aspects of consumption. We hypothesize that the individual utility derived from the use of antibiotics is weakly separable from quantities of all other types of goods consumed. Consequently, consumers follow a multistage process to allocate their budget to antibacterial products. In the first stage, the total spending is allocated to broad categories of goods such as health care versus other types of goods or services. The health care spending is then separated in subgroups such as pharmaceuticals, diagnostic tests and inpatient care. Given the nature of the infection, the budget share for pharmaceuticals is

\footnotetext{
${ }^{5} \mathrm{Ma}$ and al. (2004) estimated the share equations of animal product consumption for Chinese households. Combining the AIDS model and the two-stage Heckman procedure, Lazaridis (2004) focused on the factors affecting the demand for different types of oils and fats in Greece. Boetel and Liu (2003) estimated a meat demand system and investigated the effect of food information on the meat shares consumed.
} 
assigned to antibiotics and other types of drugs. Finally, the choice is between different categories of antibiotics according to their therapeutic attributes, the risks of bacterial resistance, and the cost of treatment. ${ }^{6}$

The individual expenditure function derived from the consumer theory is aggregated across individuals to obtain the antibiotic expenditure in the local market area. $^{7}$ Muellbauer $(1975,1976)$ showed that exact aggregation is possible within a specific family of preferences. These preferences are known as the price independent generalized logarithmic (PIGLOG) class of preferences. The PIGLOG class can be denoted by the following expenditure function, which is the minimum expenditure necessary to achieve a certain level of utility at any given price

$$
\log c(u, p)=(1-u) \log a(p)+u \log b(p)
$$

where $u$ is the level of utility, $a(p)$ and $b(p)$ represent functions of a price vector $p$. Following Deaton and Muellbauer (1980) we assume

$$
\log a(p)=\alpha_{0}+\sum_{i} \alpha_{i} \log p_{i}+\frac{1}{2} \sum_{i} \sum_{j} \lambda_{i j} \log p_{i} \log p_{j}
$$

and

$$
\log b(p)=\log a(p)+\beta_{0} \prod_{i} p_{i}^{\beta_{i}}
$$

where $\alpha_{0}, \alpha_{i}, \beta_{0}, \beta_{i}$ and $\lambda_{i j}$ are constants, and $i$ and $j$ are indexes representing different antibiotic categories.

\footnotetext{
${ }^{6}$ The Swiss National Research Programme on Antibiotic Resistance (NPR 49) reports that information about the risk of bacterial resistance reach consumers mainly through the doctor and information campaigns, since the other kind of information like newspapers are generally inaccurate. Doctors are regularly informed by regional (cantonal) health authorities and through their own experience of non-effective treatments on their patients. Leaflets are few and also not widely distributed, although some cantonal hospitals deliver guidelines to practitioners in the area. Consumers are generally aware that bacterial resistance affecting some types of antibiotics may be harmful although direct to consumers information is not satisfactory. Patients trust their doctors for a correct understanding of the resistance problem.

${ }^{7}$ Aggregation theory provides the necessary conditions under which the aggregate demand, i.e. the representation of market demand, can be treated as if it was the outcome of the decisions of a rational representative consumer. See Muellbauer (1975) and Cornes (1992) for a general discussion.
} 
Substituting for $\log a(p)$ and $\log b(p)$ in Eq. (1) we can write the cost function as

$$
\log c(u, p)=\alpha_{0}+\sum_{i} \alpha_{i} \log p_{i}+\frac{1}{2} \sum_{i} \sum_{j} \lambda_{i j} \log p_{i} \log p_{j}+\beta_{0} u \prod_{i} p_{i}^{\beta_{i}},
$$

which is linearly homogeneous in prices, provided that the following restrictions on the parameters hold

$$
\sum_{i} \alpha_{i}=1, \sum_{i} \lambda_{i j}=\sum_{j} \lambda_{i j}=0, \sum_{i} \beta_{i}=0
$$

By applying the Shephard's lemma ${ }^{8}$ and substituting afterwards the indirect utility function ${ }^{9}$ derived from Eq. (2) we obtain the expenditure share of the $i^{t h}$ group of antibiotic substances

$$
w_{i}=\alpha_{i}+\sum_{j} \gamma_{i j} \log p_{j}+\beta_{i} \log (x / P)
$$

where $x$ is the total expenditure for antibiotics in outpatient care ${ }^{10}, P$ is a price index defined by

$$
\log P=\alpha_{0}+\sum_{i} \alpha_{i} \log p_{i}+\frac{1}{2} \sum_{i} \sum_{j} \gamma_{i j} \log p_{i} \log p_{j}
$$

and

$$
\gamma_{i j}=\frac{1}{2}\left(\lambda_{i j}+\lambda_{j i}\right)
$$

The following restrictions are implied by (5) and (8)

$$
\sum_{i} \gamma_{i j}=\sum_{j} \gamma_{i j}=0, \gamma_{i j}=\gamma_{j i} \forall i, j(i \neq j) .
$$

\footnotetext{
${ }^{8}$ Demand functions in budget share form are derived from a natural logarithmic differentiation of the expenditure function with respect to prices.

${ }^{9}$ Total expenditure $x$ is equal to $c(u, p)$ for a utility maximising consumer. Hence, $c(u, p)$ can be inverted to give $u(p, x)$, the indirect utility function.

${ }^{10}$ We define $x=\sum_{i} p_{i} q_{i}$ as the total expenditure on antibiotics for respiratory infections in outpatient care, where $p_{i}$ and $q_{i}$ represent the price and the quantity for the $i^{\text {th }}$ group of antibiotics by the representative consumer.
} 
Provided that Eqs. (5), (8) and (9) hold true, the equation (6) defines a system of demand functions. These are homogeneous of degree zero in prices and total expenditure and satisfy the Slutsky symmetry. The total expenditure is then given by $\sum w_{i}=1$.

The interpretation of the demand share summarized by Eq. (6) is straightforward. Without any change in relative prices and expenditures, i.e. the second and the third terms of the right-hand side of the equation, the budget shares of different groups of antibiotics are constant. Changes in relative prices affect the demand share through the terms $\gamma_{i j}$. These capture the effect on the $i^{\text {th }}$ budget share from a $1 \%$ increase in the price of the $j^{\text {th }}$ category of goods, with $x / P$ held constant. Changes in real expenditure are taken into account by the parameter $\beta_{i}$, which is assumed equal to zero.

The share equation also underlines some basic properties of the demand function. Other things being equal, the expenditure share of each group of commodities is inversely associated with its own price and is positively related to the price of other goods. The expected sign of $\gamma_{i i}$ is then negative. On the other side, $\gamma_{i j}$ should exhibit a positive sign for any $i \neq j$ if goods are close substitutes.

\subsection{Spatial dependency and other determinants}

The demand for antibiotics may also be affected by variables other than price that account for expenditure shifts. For instance, the incidence of infections may imply seasonal trends in the per capita consumption. The socioeconomic characteristics of a population and other aspects of health care supply may also affect antibiotic use. These aspects may be of little relevance in the demand share of different classes of antibiotics, unless they shape preferences for specific antibiotic categories.

It has been suggested that the patients' age significantly affects the route of administration of antibiotics for lower respiratory tract infections (Mazzaglia et al., 1999) and the type of antibiotic used (Pendergrast and Marrie, 1999). Moreover, physicians may have an incentive to prescribe the newer and more expensive antibi- 
otics, that is ones with a broader spectrum of activity, which apparently reduces risks for patients. Our model can be easily modified to account for expenditure shifts determined by the structure of the population and cultural differences. ${ }^{11}$

Following Ray (1980) and Pollak and Wales (1992), we extend our model to include the additional determinants by a log-linear scaling procedure. Cultural differences across regions are taken into account by dummy variables.

From Eq. (6) the AIDS model can then be extended to analyse aggregate data on antibiotic consumption across small geographic areas. Assuming separability in quantities consumed for different types of goods, we can write the econometric specification as

$$
\begin{aligned}
w_{i}= & \alpha_{i}+\sum_{j} \gamma_{i j} \log p_{j}+\beta_{i} \log (x / P)+\sum_{k=1}^{S} \nu_{i k} \log V_{k}+\psi_{i} \log H+ \\
& +\phi_{i} \log R_{i}+\sum_{t=1}^{T} \rho_{i t} D T_{t}+\theta_{i} M w_{i}+u_{i},
\end{aligned}
$$

where $\nu_{i k}, \psi_{i}, \phi_{i}, \rho_{i t}$ and $\theta_{i}$ are new parameters. The index $i=1,2,3,4$ denotes four groups of antibiotic substances discussed later on in section 3 and summarized in table 1.

From the econometric point of view, the choice of the appropriate method for the estimation of Eq. (10) should consider the possibility of spatial autocorrelation in antibiotic consumption. We found evidence of spatial autocorrelation using the Moran's I test. We then modified the extended AIDS model to take spatial aspects into account. To our knowledge, the economic literature lacks extended AIDS models with spatial effects and the approach has not been previously applied to the consumption of drugs. ${ }^{12}$ We introduced spatial lags of consumption in the

\footnotetext{
${ }^{11}$ Socioeconomic determinants of outpatient antibiotic use in Switzerland have been investigated in previous studies by Filippini et al. (2006, 2009). The authors apply multivariate parametric approaches to the aggregate demand of antibiotics but fail to investigate determinants of different antibiotic groups.

${ }^{12}$ Spatial dependency in AIDS models has been applied, for instance, by Paraguas et al. (2006) to investigate fish expenditure in the Philippines. The model, however, does not include socioeconomic factors as determinants of consumption.
} 
econometric specification following the spatial autoregressive parameter approach proposed by Anselin (2001). We added spatial lags of consumption, $\theta_{i} M w_{i}$, by means of the spatial weight matrix $M{ }^{13}$ The consumption of antibiotic group $i$ in one area is assumed to be affected by the level of consumption in neighbouring areas. Indeed, spatially adjacent areas may exhibit similar behaviour in terms of antibiotic utilization due to inherent common spatial influences.

The econometric specification in Eq. (10) includes a set of variables, $V_{k}$, that captures the demographic and cultural characteristics of the population. $H$ is a proxy for the health status of the population represented by the incidence of main bacterial infections at the cantonal level, and $R_{i}$ are indicators of bacterial resistance. ${ }^{14}$ The magnitude of bacterial resistance country-wide and for macro regions is known to doctors and patients, but such a figure is not broken down for smaller areas. We operate on the plausible assumption that bacterial resistance varies across macro regions rather than across smaller areas. However, given the information available, the doctor's as well as the patient's attitude towards the risk perception of bacterial resistance may differ across regions. Physicians and patients may then have a heterogeneous attitudes towards the use of different types of antibiotics. Differences in the perception of the resistance problem may be related, for instance, to cultural aspects included in $V_{k}$.

Finally, $D T_{t}(t=1, . ., 3)$ are time dummies, and the random term $u_{i}$ is normally

\footnotetext{
${ }^{13}$ The modified version of the AIDS model that uses spatial lags is built on the structure of Dynamic Almost Ideal Demand Systems. The way to introduce dynamic elements into the demand system analysis is discussed, for instance, by Gracia et al. (1998) and Shukur (2002). The spatial lag can be thought of as an "inertia lag" in the sense that consumers slowly adjust their consumption behaviour with the neighbouring areas. Consequently, spatial dependency can be introduced by means of an autoregressive specification in the demand system equations.

14 Indicators of bacterial resistance are defined as ratios between the number of non-susceptible bacteria isolates over the number of tested isolates in regional laboratories and reported in regional hospitals guidelines. We built five types of indicators: susceptibility of Staphylococcus aureus (SA), susceptibility of Streptococcus pneumoniae (SP), susceptibility of Escherichia coli (EC), susceptibility of Campylobacter jejeuni (CJ) and susceptibility of all types of bacteria (ALL), which is an average of the above indicators. Since each type of bacteria is tested against different antibiotic substances, we built susceptibility indicators for each antibiotic group by averaging the results of susceptibility tests to main substances in the group.
} 
and identically distributed with variance $\sigma_{\varepsilon}^{2}$.

The expenditure share function is linearly homogeneous in all the explanatory variables provided that the new parameters satisfy the following condition:

$$
\sum_{i} \nu_{i k}=\sum_{i} \psi_{i}=\sum_{i} \phi_{i}=\sum_{i} \rho_{i t}=\sum_{i} \theta_{i}=0
$$

and $P$ is approximated by the Stone's geometric price index

$$
\log P=\sum_{i} w_{i} \log \left(p_{i}\right)
$$

To avoid simultaneity problems, we use the median values of the expenditure shares to calculate the Stone price index. Moreover, to reduce the risk of endogeneity generated by the fact that prices of different groups of antibiotics are computed using antibiotic shares, we consider lagged values for prices.

\subsection{Expenditure and price elasticities}

Since we are interested in studying the response of the demand for different antibiotic groups to changes in price and expenditure, we calculate elasticities at the sample mean of expenditure shares. Using Eqs. (10) and (12) we derive the uncompensated (Marshallian) own-price elasticities $\left(\varepsilon_{i i}\right)$ and cross-price elasticities $\left(\varepsilon_{i j}\right) \operatorname{as}^{15}$

$$
\begin{aligned}
\varepsilon_{i i} & =-1+\frac{\gamma_{i i}}{w_{i}}-\beta_{i}, \\
\varepsilon_{i j} & =\frac{\gamma_{i j}}{w_{i}}-\beta_{i} \frac{w_{j}}{w_{i}}, i \neq j .
\end{aligned}
$$

Using the Slutsky equation, we then obtain the expenditure elasticity for the $i^{\text {th }}$ antibiotic category given by

$$
\eta_{i}=1+\frac{\beta_{i}}{w_{i}}
$$

A positive value suggests that good $i$ is normal.

\footnotetext{
${ }^{15}$ See Alston et al. (1994) and Chalfant (1987) for details.
} 
The income compensated or net (Hicksian) own-price elasticities $\left(\delta_{i i}\right)$ and crossprice elasticities $\left(\delta_{i j}\right)$ are obtained by applying the Slutsky decomposition to Eq. (15) and using the price index in (12). These can be written as

$$
\begin{gathered}
\delta_{i i}=-1+\frac{\gamma_{i i}}{w_{i}}+w_{i}, \\
\delta_{i j}=\frac{\gamma_{i j}}{w_{i}}+w_{j}, i \neq j .
\end{gathered}
$$

Consumer theory suggests that compensated own-price elasticities are negative for normal goods. Moreover, if Eqs. (14) and (17) are positive the two groups of antibiotics are cross substitutes, otherwise they are complements.

Using the Slutsky equation once again, it is possible to derive a relationship between the compensated cross-price elasticities and expenditure elasticities: $\varepsilon_{i j}=$ $w_{j} \sigma_{i j}-w_{j} \eta_{i}$, where $\sigma_{i j}$ are the partial elasticities of substitution, known also as the Allen elasticities of substitution

$$
\sigma_{i j}=1+\frac{\gamma_{i j}}{w_{i} w_{j}} i \neq j
$$

The sign of $\sigma_{i j}$ determines whether the goods $i$ and $j$ are complements or substitutes. If $\sigma_{i j}$ is positive (negative) the two goods are substitutes (complements).

\section{Data and variables}

Our data covers 240 contiguous market areas that represent the entire Swiss territory. The areas exhibit a good degree of internal homogeneity in terms of population density and providers of health care. Aggregate data on outpatient antibiotic sales of different classes of antibiotics were provided by IHA-IMS Health Market Research. ${ }^{16}$ The dataset is detailed at product/brand name and data are available quarterly for

\footnotetext{
${ }^{16}$ Data on antibiotic sales derived from transactions between wholesalers and pharmacies and physicians. Clearly, we are aware that the quantity of antibiotics sold and the quantity actually consumed may differ. In this study we assume that patient non-compliance with doctor's prescription, if any, is a negligible factor. Finally, the potential mismatch between wholesale records and prescribing data due to seasonal fluctuations of retailer's stocks is assumed to have only a limited temporary effect and is likewise ignored.
} 
2002. Since we focus on respiratory infections, we limited our analysis to the antibiotics mainly used in the treatment of these diseases. However, part of these antibiotics can also be used for the treatment of other types of infections like gastrointestinal infections. We aggregated active substances according to the antibiotic class and the generation. Consequently, we kept $66 \%$ of the original dataset in terms of sales. We excluded antibiotics with a parenteral route of administration. Four antibiotic groups were identified (classic penicillins, penicillins amoxi/clav plus 1st and 2nd generation of cephalosporins, 3rd generation of cephalosporins and quinolones, as well as macrolides) using the relevant literature on antibiotic treatments and suggestions by experts. These groups are summarized in Table 1.

[Table 1: about here]

Penicillins are among the first discovered antibiotics and have been largely used against $S$. pneumoniae pathogens, which are a primary cause of respiratory tract infections (Schito et al., 2000). In particular, classic penicillins (penicillins V, ampicillin, amoxicillin) are commonly utilized to treat angina from streptococci and only rarely for other types of respiratory infections. The rate of penicillin-resistance $S$. pneumoniae has substantially increased since the mid-1980s (CDC, 1994).

Newer penicillins (amoxi/clav) can be combined with some cephalosporins since they belong to the same broad classification (beta-lactams). The spectrum of amoxi/clav and 2nd generation of cephalosporins are very similar. Observed crossresistance between cephalosporins and penicillins may imply that patients infected by penicillin-resistant bacteria are resistant to cephalosporins as well. The 1st generation of cephalosporins is currently of very little use.

The 3rd generation of cephalosporins has a broader activity spectrum compared to the previous generations, which implies that it can be used against a larger variety of bacteria. Consequently, it is suitable for more severe infections. Nevertheless, it is generally used as an alternative to the 2 nd generation. Practices may significantly 
vary across the areas since some doctors are more likely than others to preserve the 3rd generation for more resistant bacteria.

Similarly, quinolones have a large range of antibacterial activity, which includes multidrug resistant strains responsible for respiratory tract infections. Since this category should also be used with caution and better preserved for more severe cases, we considered it in a group together with the 3rd generation of cephalosporins. When the nature of the infection is uncertain and doctors are quite risk averse, 3rd generation of cephalosporins and quinolones may be preferred to penicillins and other cephalosporins.

Finally, macrolides are generally known as an alternative to beta-lactams. In some cases, bacterial resistance is more severe than for penicillins. Resistance to macrolides has increased over time among penicillin-resistant pneumococci and penicillin-susceptible strains. The preference for either penicillins or macrolides may then depend upon established local practices and patients preferences.

Average prices of each antibiotic group have been imputed using expenditure data and quantities. Quantities are measured in days of treatment (DOT) and prices are consequently defined in currency units per one day of treatment. A daily dose is standardised by the (WHO) World Health Organisation.

[Table 2: about here]

Table 2 gives summary statistics of expenditure shares and prices for the four groups of antibiotics. Penicillins (amoxi/clav) and early generations of cephalosporins and macrolides represent the largest shares of the expenditure. Note, however, that strong differences are observed across the areas. These categories may account for only $15 \%$ of the total expenditure. On the other hand, in some areas they are largely used and may reach a share of more than $70 \%$.

Information on the other variables included in the model (see Table 3) are mainly obtained from IHA-IMS . Demographic variables include the share of the population 
classified in five ranges of age. Note, for instance, that individuals under 26 represent less than $1 \%$ of the total population in some areas and more than $20 \%$ in others. Similarly, the proportion of people between 60 and 74 largely varies across areas, from $9.5 \%$ to almost 25\%. French and Italian speaking areas represent $43.7 \%$ of all areas and are generally characterized by a more intensive use of antibiotic per capita. We defined a dummy variable that takes value equal to 1 if the area has a Latin culture (French and Italian) and 0 otherwise.

[Table 3: about here]

The data on health status of patients is based on information on the incidence of respiratory and common gastrointestinal diseases. As indicators we use the incidence of $S$. pneumoniae infections, which represent the most common airborne bacterial infections among the population. We alto take the incidence of Campylobacter and Salmonella infections, which are a leading cause of gastrointestinal infections. The reason for including data on gastrointestinal infections is that these data are generally more reliable than those for airborne bacterial infections because of availability of information for all regions, larger sample, and reduced risk of bias. ${ }^{17}$ Although in most cases a medical treatment is required to recover from these infections, patients, especially children and old people, may be prescribed an antibiotic when symptoms are particularly severe.

Finally, the magnitude of bacterial resistance is calculated for three macro regions. There is a lack of resistance indicators for outpatient antibiotics covering the Swiss territory. A systematic collection of data had not started until recently. However, regional hospital guidelines include a summary of resistance indicators and are generally prepared every two years and are available to general practitioners. The indicators are provided by regional laboratories that collect samples of bacteria isolates from different settings like hospital wards and out of doors and test them

\footnotetext{
${ }^{17}$ Our information comes from yearly publications of the Swiss Federal Statistical Office at the cantonal level.
} 
against several antibiotic substances. To construct our indicators we took the average result of tests for antibiotic substances representative of each antibiotic category and published between 1999 and 2003. Following the SEARCH (Sentinel Surveillance of Antibiotic Resistance in Switzerland) project standard, we considered three macro regions: east, west and mid. Indicators for the three regions were then obtained from main regional hospitals: Bern (mid), Geneva (west), Lugano (east), and Zurich (east). Since Lugano and Zurich belong to the same region (east), we averaged indicators from these hospitals guidelines to derive a resistance indicator for the east region. Although the information on antibiotic resistance could be broken into smaller areas, we believe that this is one of the first attempts to include resistance indicators in econometric models of antibiotic consumption. ${ }^{18}$

In our regressions we test five susceptibility indicators: Staphylococcus aureus, $S$. pneumoniae, Escherichia coli, Campylobacter jejeuni, and a mix indicator (average of the above indicators). In Table 3 we report the susceptibility of different antibiotic groups to the $S$. pneumoniae which is used as an indicator in the basic specification of the model. The reason is that $S$. pneumonie is more accurately related to respiratory infections than other bacteria and the impact of the combined indicator is not significantly different. The ranking between antibiotic groups in terms of bacterial susceptibility is not straightforward since some types of bacteria are more likely to be susceptible to specific antibiotic substances. ${ }^{19}$

\footnotetext{
${ }^{18}$ Local areas in Switzerland are by far smaller than geographical areas usually investigated in many other countries. It is implausible that bacterial resistance varies significantly across such small areas. Health authorities are responsible for larger areas (cantons). Although we distinguish between "regional" and "local" information on bacterial resistance, the latter are neither available nor are there plans to make them available in the near future. Clearly, there may be a margin for improvement since we rely on information available for three macro areas which represent an aggregation of cantons. However, some cantons are indeed very small and may not be able to provide statistically significant data for the surveillance system. For this reason, the sentinel surveillance system collects and publishes resistance data for macro regions.

${ }^{19}$ Also for this reason we consider several resistance indicators. However, the estimation results do not differ significantly. In particular, estimations with susceptibility of Streptococcus pneumoniae and the average susceptibility to all bacteria exhibit very similar figures. The main results of the model are also confirmed without resistance indicators.
} 


\section{Estimation results}

The spatial AIDS model defined by Eq. (10) is used to investigate the expenditure shares of different groups of antibiotics. Explanatory variables include the price of antibiotics, population characteristics like age structure and income and cultural aspects, the incidence of community-acquired infections, and seasonal dummies. In addition, the share of different antibiotic groups can be influenced by the level of bacterial resistance and antibiotic consumption in adjacent areas.

We estimate the model through the Zellner's Iterative Seemingly Unrelated Regression (SUR) procedure with the software STATA. The set of restrictions leads to a singular residual variance/covariance matrix. Consequently, we drop one share equation from the system. This is the first group (classic penicillins) which represents the smallest budget share on average across the four groups. Using the estimated parameters of the share equations of the other three groups and the restrictions applied above, we then obtain the parameters for the dropped group.

The estimation results are reported in Table 4. Each equation is estimated with 720 observations, since data for 240 areas are quarterly available, and one quarter is excluded to allow for the use of lagged values for prices. The adjusted $\mathrm{R}^{2}$ suggests that selected variables explain approximately $36 \%, 40 \%$ and $48 \%$ of cross-area variations in the use of antibiotics, respectively for group 2 (penicillins amoxi/clav and early generations of cephalosporins), group 3 (3rd generation of cephaloporines and quinolones) and group 4 (macrolides). ${ }^{20}$

The estimated spatial autoregressive parameter associated with the lag term $D I D_{-i}$ is significant and positive. ${ }^{21}$

\footnotetext{
${ }^{20}$ The adjusted $\mathrm{R}^{2}$ reported in our estimations are satisfactory considering that the dataset is dominated by cross-sectional variations, hence we are mainly explaining cross-sectional variation in antibiotic consumption. For a general discussion of the interpretation of the $\mathrm{R}^{2}$ in AIDS models see Verbeek (2008).

${ }^{21}$ The result suggests the evidence of consumption externalities across the areas. Higher consumption of one group of antibiotics in one area is significantly associated with higher consumption of that group in adjacent areas. A plausible explanation for this result lies in the perceptions of doctors and patients of the antibiotic group that is the most appropriate for the treatment of common respiratory infections. Preferences may be affected by practice styles in adjacent areas.
} 
[Table 4: about here]

The impact of consumer expenditure on the demand share of group 2 is negligible, and negative for groups 3 and 4 . Note, however, that the coefficients are never significant. As detailed in Table 5 below, income elasticities are all positive, which suggests that different outpatient antibiotic groups are normal goods.

Most price coefficients are significant at less than $1 \%$ with few exceptions, but the demand for group 3 and group 4 seems to be positively related to their own prices and negatively related to the price of other groups. Price elasticities will be analysed later on in section 4.1 as we investigate complementary and substitution effects between antibiotic groups.

The impact of demographic variables and cultural aspects on the demand share of all the four antibiotic groups is plausible but coefficients are not highly significant. The age class $26-59\left(\mathrm{POP}_{3}\right)$ is the baseline category. The proportion of individuals over 74 years of age $\left(\mathrm{POP}_{5}\right)$ reflects some health considerations. The coefficient is positive and significant at less than $10 \%$ for antibiotic group 3, whereas it is negative and significant at less than $1 \%$ for antibiotic group 4 . This may suggest that elderly people are more exposed to the risk of severe infections in the community as compared to other individuals and these infections imply greater health risks. Indeed, antibiotics in group 3 (3rd generation of cephalosporins and quinolones) are commonly used for severe cases. The literature on the determinants of health care expenditure indicates that the increasing prevalence of chronic health problems associated with aging may cause an increase in the utilization of health care services (Di Matteo and Di Matteo, 1998; Di Matteo and Grootendorst, 2002). We argue that the share of antibiotics for more severe infections may increase with the proportion of elderly patients in the community. Our result is also in accordance with findings by Mazzaglia et al. (1999), who suggested that cephalosporins are the antibiotics most frequently prescribed for lower respiratory tract infections in adults, while macrolides are the less prescribed ones. 
We did not find clear evidence that a higher proportion of children $\left(\mathrm{POP}_{1}\right)$ negatively affects the share of antibiotic group 1 (penicillins) and positively affects the share of macrolides. According to Otters et al. (2004), there has been a decrease in the proportion of narrow-spectrum antibiotics prescribed for children between 1987 and 2001 in the Netherlands. By contrast, the proportion of macrolides increased from $8 \%$ to $16 \%$. Similarly, Schindler et al. (2004) showed that macrolides were the most frequently prescribed antibiotics (48.1\%) for respiratory infections among children aged between 0 and 6 in Germany.

Areas with a French/Italian culture exhibit a significantly lower consumption of macrolides (group 4) and a higher consumption of 3rd generation cephalosporins and quinolones (group 3) compared to areas with a German culture. Similar differences are observed at a larger scale across European countries. France and Italy, for instance, use a relatively larger amount of 3rd generation cephalosporins as compared to other European countries (Coenen et al. 2006).

The coefficient of the incidence of infections significantly increases with the share of antibiotics in group 2 and negatively affects antibiotics in group 3 . The result hypothesizes that epidemiological differences are relevant in explaining variations in the use of different types of antibiotics in the community. An increase in the spread of community-acquired infections raises the share of narrow-spectrum antibiotics rather than the share of the latest generation of antibiotics more suitable for severe cases.

The study of the impact of bacterial resistance on the utilization of different antibiotic groups may add some insights to the analysis of epidemiological aspects although the interpretation needs a lot of carefulness. We did not find evidences to suggest that the share of different antibiotic groups is affected by the degree of bacterial susceptibility in the area, except for group 3. There may be some plausible explanations for this result. Since data are available for macro regions through hospital guidelines, information may only partially become available to consumers. Guidelines may not reach general practitioners and patients effectively. The extent to which information is correctly understood and utilised cannot be assessed. 
Therefore, the interpretation of the relationship between bacterial resistance and antibiotic demand in the following section could be undermined by this limitation.

Finally, we find remarkable seasonal effects on antibiotic shares. The baseline season is represented by the fourth quarter, i.e. the autumn dummy (October, November, December). The consumption of more expensive antibiotics (group 3 and 4) is higher in winter (1st quarter) and lower during the summer (3rd quarter). This may connote higher risk aversion of doctors and patients in periods when the incidence of infections is higher and severe infections occur more frequently. Accordingly, the patient's willingness to pay for drugs may rise with the perceived risk of infection. By contrast, less expensive antibiotics, such as penicillins and early generations of cephalosporins, are used less often during the first quarter and more often in the summer quarter.

\subsection{Elasticities}

Using the estimation results from Table 4 and applying the definitions derived in section 2.2, we calculate the own-price, cross-price and expenditure elasticities of the demand for different antibiotic groups. The figures are summarized in Table 5 and Table 6 . Some important implications can be straightforwardly derived. ${ }^{22}$

[Table 5: about here]

Expenditure elasticities are positive for all antibiotic groups. The result may suggest that antibiotics are normal goods and is in accordance with Baye et al. (1997), who estimated that anti-infectives have positive income elasticity (around 1.3). More specifically, Chaudhuri et al. (2003) observed positive expenditure elasticities for different types of quinolones, ranging from 0.3 to 2.20 . Since our expenditure

\footnotetext{
${ }^{22}$ As mentioned above, the demand system is estimated by assuming separability between the consumption of antibiotics and other goods. The implication of this assumption is that the elasticities are partial elasticities, for example they are conditional upon allocation of total expenditure between antibiotics and other goods.
} 
elasticities are very close to one, the consumption share of each group of antibiotics remains constant as the amount spent on other groups changes. The evidence then indicates that antibiotics in outpatient care can be denoted as necessities. As income rises, the need for additional consumption of antibiotics is negligible, ceteris paribus.

Grootendorst and Levine (2006) showed that pharmaceutical demand elasticities depend on whether patients receive social assistance, or non-eligible patients have private health insurance. Health insurance in Switzerland is mandatory for residents and the same basic contract is offered by competing private insurance companies. The insurance premium varies according to a limited menu of deductibles. The individual's income and health status partially affect the selected level of deductible.

All uncompensated own-price elasticities have the expected negative sign and vary substantially from -0.636 to -1.005 . The comparison with the existing literature is not straightforward, although some similarities may be pointed out. Focusing on anti-infectives, Baye et al. (1997) estimated uncompensated own-price elasticity equal to -0.916. Ellison et al. (1997) found significant and negative uncompensated own-price elasticities for the four types of cephalosporins analysed. Looking at quinolones only, Chaudhuri et al (2003) calculated a relatively high uncompensated own-price elasticity, equal to -2.5 on average and ranging from -0.45 to -5.5 .

[Table 6: about here]

Our estimates indicate that the demand for 3rd generation cephalosporins and quinolones (group 3) and the demand for classic penicillins (group 1) are more price elastic than the demand for penicillins amoxi/clav and 1st and 2nd generation cephalosporins (group 2) and the demand for macrolides (group 4). It is worth noticing that the highest own-price elasticities are found for the most expensive antibiotic category (group 3) and the traditional and less frequently used antibiotics (classic penicillins). The rationale may be that doctors and patients are more likely 
to increase or reduce the consumption of the latest generation of cephalosporins and quinolones when their price changes, since this category of antibiotics is at least partially used to reduce uncertainty on the severity of the infection and the risks of bacterial resistance. On the other hand, they are less likely to leave commonly used antibiotic therapies such as previous generations of cephalosporins, penicillins and macrolides. Classic penicillins represent a traditional antibiotic therapy whose comparative advantage has been substantially undermined by better alternatives. Consequently, they may be quite sensitive to variations in relative prices.

As expected, the Hicksian own-price elasticities are smaller in magnitude compared to the Marshallian elasticities. This indicates that the pure effect of substitution is only partially compensated by the income effect.

Substitution and complementary relationships among antibiotic groups are captured by the Allen elasticities summarized in Table 6. Positive values denote that the two groups are substitutes. In our case, narrow spectrum antibiotics such as penicillins amoxi/clav and early generations of cephalosporins (group 2) do not appear to be good substitutes for antibiotics with a larger spectrum such as new cephalosporins and quinolones (group 3). The rationale may be that old generations of cephalosporins are not perceived to be effective against severe infections. Moreover, newer generations are generally taken into account to overcome specific problems of bacterial resistance encountered in the use of previous generations.

When the price of group 1 (classic penicillins) and group 4 (macrolides) increases, doctors may prefer to switch to penicillins amoxi/clav and cephalosporins (group 2) rather than to more effective antibiotics such as newer generations of cephalosporins and quinolones (group 3). Elasticities of substitution between groups 1 and 4 and groups 2 and 3 are positive.

The Allen's elasticity of substitution confirms that the demand for classic penicillins follows the demand for macrolides when the price of the latter category changes. The result may be associated with a certain degree of persistency in the patient's tastes. For instance, doctors argue that children have a preference for macrolides rather than penicillins. This could imply that an increase in the price 
of macrolides is likely to induce a higher consumption of cephalosporins or newer antibiotic components, rather than drive the utilization of classic penicillins upward, ceteris paribus.

\section{Conclusion}

Antibiotic misuse and the cost of bacterial resistance are major threats to efficient treatment against community-acquired respiratory infections. The demand for antibiotics has been investigated in studies by Ellison et al. (1997) and Chaudhuri et al. (2003). Their focus is on specific market segments where antibiotic substances constitute close therapeutic substitutes and are similar in terms of the impact of bacterial resistance. There are, however, arguments for considering a more extended set of drugs. The major contribution of this study is the investigation of sources of local variations in the use of different types of antibiotics in the community using an econometric approach that takes into account the problem of spatial autocorrelation. For this reason, we included a spatial lag on antibiotic consumption in the linear approximate demand system model to investigate antibiotic consumption across small areas. Moreover, we added several socioeconomic determinants of antibiotic use in the model, such as demographic and cultural characteristics of the population and the patients' health status. Therapeutic components are aggregated in four groups suitable to define, to the best of our knowledge, the doctor's and patient's choice set in the treatment of community-acquired respiratory infections in Switzerland. Estimations are carried out on 2002 quarterly data of defined daily doses per 1000 inhabitants (DID) available for 240 small areas.

The results lead us to the conclusion that the highest price demand elasticities are observed for the 3rd generation cephalosporins and quinolones, which are the newer and more expensive antibiotic category; and classic penicillins, which are the most traditional and cheaper category. We found complementary effects between antibiotics with a relatively narrow spectrum (penicillins amoxi/clav and cephalosporins 1st/2nd generations) and antibiotics with a relatively large spectrum 
(3rd generation cephalosporins and quinolones), and between classic penicillins and macrolides. The demand elasticity suggests a good degree of substitution between the other groups, for instance between penicillins amoxi/clav and cephalosporins 1st/2nd generations (group 2) and macrolides (group 4).

Looking at the impact of population characteristics, we observed that an increasing proportion of elderly people positively affects the proportion of new cephalosporins/quinolones (group 3) and reduces the proportion of macrolides used. The rationale behind this may be that elderly people are more exposed to severe infections and to the risk of bacterial resistance. Italian- and French-speaking regions are associated with a more substantial use of newer and more expensive antibiotics (group 3) and a lower proportion of macrolides (group 4).

Because different groups of antibiotics are associated with different degrees of bacterial resistance, variations in the practice of physicians and in the patients' attitudes are probably related to the stance towards the risk of bacterial resistance. If bacterial resistance is developed for a certain class of antibiotics, no amount of cross elasticity between this class and another class may persuade patients to purchase or doctors to prescribe the ineffective class. Our approach makes an attempt to take this aspect into account. Ignoring the possibility of awareness of bacterial resistance by the general public would leave some ambiguities in our model. Our analysis addresses the consumer's willingness to trade off a lower level of bacterial resistance to a relatively higher price, since we tested the impact of available information on bacterial resistance. Some evidence was found for one antibiotic group (new cephalosporins/quinolones) only. This points at possible limitations of our data. Firstly, data on bacterial resistance are available for macro regions only rather than for local areas. Note, however, that relevant information on bacterial resistance in Switzerland is meaningful at regional level. Although variations across smaller areas are plausibly of little relevance and, consequently, not monitored, current indicators are built on a limited number of sources. Secondly, information on bacterial resistance may not be widely disseminated since it derives from guidelines prepared by regional hospitals and are not always delivered to practitioners and 
consumers. Finally, our resistance indicators may not fully reflect the effectiveness of antibiotic groups that combine different antibiotic substances. Clearly, we cannot verify whether the dissemination of resistance data is effective and patients are fully aware of the resistance problem even though collected data are reliable and resistance indicators are easy to understand since they are provided in the form of simple ratios.

Despite limitations, the analysis helps to enlighten the impact of determinants of variations in the use of different types of antibiotics in the community. Our findings indicates that health care policy makers may improve efficiency in antibiotic use by taking economic incentives into account. One example can be a more effective dissemination of information on prices and the impact of bacterial resistance to physicians and patients. The Swiss Sentinella on antimicrobial resistance relies on a network of laboratories in the country and publishes resistance indicators on the web. The collection of data and the provision to the Sentinella can be eased by the implementation of a national monitoring centre, to be appointed by the federal authority. The information provided could then be used by regional health authorities to prepare leaflets and publish articles in newspapers targeted at the general public and/or general practitioners. Finally, the dissemination of information could be combined with another policy instrument like a regional tax/subsidy system that induces the use of antibiotic categories less affected by resistance problems or antibiotics (generally those with narrow spectrum) that are less likely to contribute to the resistance problem. 


\section{References}

Alston JM, Foster KA and Green RD, "Estimating elasticities with the Linear Approximate Almost Ideal Demand System: some Monte Carlo results", The review of Economics and Statistics, 76, 2, 351-356, 1994.

Alvarez-Elcoros S and Enzler MJ, "The macrolides: Erythromycin, clarithromycin, and azithromycin", Mayo Clinic Proceedings, 74, 6, 613-634, 1999.

Anselin L, "Spatial Econometrics", in: Baltagi B, A Companion to Theoretical Econometrics, Oxford: Basil Blackwell, 310-330, 2001.

Baye MR, Maness R and Wiggins SN, "Demand systems and the true subindex of the cost of living for pharmaceuticals", Applied Economics, 29, 1179-1189, 1997.

Blasi F, Tarsia P, Aliberti S, Santus P and Allegra L, "Highlights on the appropriate use of fluoroquinolones in respiratory tract infections", Pulmonary Pharmacology and Therapeutics, 19, 11-19, 2006.

Boetel BL and Liu DJ, "Evaluating the effect of generic advertising and food health information within a meat demand system", Agribusiness, 19, 3, 345-354, 2003.

Centers for Disease Control and Prevention (CDC), "Prevalence of penicillin-resistant streptococcus pneumoniae - Connecticut, 1992-1993", Morbidity and mortality weekly report 43, 12; 216-217, 1994.

Chalfant J, "A global Flexible, Almost Ideal Demand System", Journal of Business and Economic Statistics, 5, 233-242, 1987.

Chaudhuri S, Goldberg PK and Jia P, "Estimating the effects of global patient protection in pharmaceuticals: a case study of quinolones in India", Working paper 10159, National Bureau of Economics Research, 2003.

Cockburn J and Pit S, "Prescribing behaviour in clinical practice: patients' expectations and doctors' perceptions of patients' expectations - a questionnaire study", British Medical Journal, 315, 520-523, 1997.

Coenen S, Ferech M, Dvorakova K, Hendrickx E, Suetens C and Goossens H, "European Surveillance of Antimicrobial Consumption (ESAC): outpatient cephalosporin use in Europe", Journal of Antimicrobial Chemotherapy, 58, 413-417, 2006. 
Cornes R, "Duality and Modern Economics", Cambridge: Cambridge University Press, 1992.

Deaton A and Muellbauer J, "An almost ideal demand system", American Economics Review, 70, 312-326, 1980.

Di Matteo L and Di Matteo R, "Evidence on the determinants of Canadian provincial government health expenditures: 1965-1991", Journal of Health Economics, 17, 211228, 1998.

Di Matteo L and Grootendorst P, "Federal patent extension, provincial policies, and drug expenditures, 1975-2000", Canadian Tax Journal, 50, 6, 1913-1948, 2002.

Ednie LM, Visalli MA, Jacobs MR, and Appelbaum PC, "Comparative activities of clarithromycin, erythromycin, and azithromycin against penicillin-susceptible and penicillin-resistant pneumococci", Antimicrobial Agents and Chemotherapy, December, 40, 8, 1950-1952, 1996.

Ellison SF and Hellerstein JK, "The economics of antibiotics: an exploratory study", In: Triplett J, ed., Measuring the prices of medical treatments, Washington, DC: Brookings Institution Press, 118-43, 1999.

Ellison SF, Cockburn I, Griliches Z and Hausman J, "Characteristics of demand for pharmaceutical products: an examination of four cephalosporins", Rand Journal of Economics, 28, 3, 426-446, 1997.

Filippini M, Masiero G and Moschetti K, "Socioeconomic determinants of regional differences in outpatient antibiotic consumption: Evidence from Switzerland", Health Policy, 78, 77-92, 2006.

Filippini M, Masiero G and Moschetti K, "Small area variations and welfare loss in the use of outpatient antibiotics", Health Economics, Policy and Law, 4, 55-77, 2009.

Gracia A, Gil JM and Angulo AM, "Spanish food demand: a dynamic approach". Applied Economics, 30, 1399-1405, 1998.

Gonzalves R, Bartlett JG, Besser RE, Cooper RJ, Hickner MJ, Hoffman JR, and Sande MA, "Principles of Appropriate Antibiotic Use for Treatment of Acute Respiratory Tract Infections in Adults: Background, Specific Aims, and Methods", 
Annals of Internal Medicine, 134, 6, 479-486, 2001.

Grootendorst PV and Levine M, "Do Drug Plans Matter? Effects of Drug Plan Eligibility on Drug Use Among the Elderly, Social Assistance Recipients and the General Population". Social and Economic Dimensions of an Aging Population Research Papers 73, McMaster University, 2002.

Howard DH, "Resistance-induced antibiotic substitution", Health Economics, 13, 585-595.

Kern WV, de With K, Nink K, Steib-Bauert M and Schröder H, "Regional variation in outpatient antibiotic prescribing in Germany". Infection, 34, 5, 269-273, 2006.

Lazaridis P, "Olive Oil Consumption in Greece: A Microeconometric Analysis", Journal of Family and Economic Issues, 25, 3, 411-430, 20042004.

Laxminarayan R and Weitzman ML, "On the implications of endogenous resistance to medications", Journal of Health Economics, 21, 4, 709-18, 2002.

Ma H, Rae A, Huang J and Rozelle S, "Chinese animal product consumption in the 1990s", The Australian Journal of Agricultural and Resource Economics, 48, 4, 569-590, 2004.

Mazzaglia G, Arcoraci V, Greco S, Cucinotta G, Cazzola M and Caputi AP, "Prescribing habits of general practitioners in choosing an empirical antibiotic regimen for lower respiratory tract infections in adults in Sicily", Pharmacological Research, 40, 1, 47-52, 1999.

Muellbauer J, "Aggregation, income distribution and consumer Demand", The Review of Economic Studies, 62, 525-43, 1975.

Muellbauer J, "Community preferences and the representative consumer", Econometrica, 44, 976-99, 1976.

Otters HB, van der Wouden JC, Schellevis FG, van Suijlekom-Smit LW and Koes BW, "Trends in prescribing antibiotics for children in Dutch general practice", Journal of Antimicrobial Chemotherapy, 53, 361-366, 2004.

Paraguas FJ, Dey MM and Kamil AA, "Spatial linear approximate to almost ideal demand system", Advances and Applications in Statistics, 6, 3, 361-375, 2006.

Pendergrast J and Marrie T, "Reasons for choice of antibiotic for the empirical treat- 
ment of community acquired pneumonia by Canadian infectious disease physicians", Canadian Journal of Infectious Disease, 10, 4, 337-345, 1999.

Pollak RA and Wales TJ, "Demand system specification and estimation", New York: Oxford University Press, 1992.

Ray R, "Analysis of a time series of household expenditure surveys for India", The Review of Economics and Statistics, 62, 4, 595-602, 1980.

Rowthorn R and Brown GM, "Using antibiotics when resistance is renewable", in Battling resistance to antibiotics and pesticides, Laxminarayan R, ed., RFF press, 2003.

Schindler C, Krappweis J, Morgenstern I and Kirch W, "Prescriptions of systemic antibiotics for children in Germany aged between 0 and 6 years", Pharmacoepidemiology and Drug Safety, 12, 113-120, 2003.

Schito GC, Debbia EA and Marchese A, "The evolving threat of antibiotic resistance in Europe: new data from the Alexander Project", Journal of Antimicrobial Chemotherapy, 46, 3-9, 2000.

Shukur G, "Dynamic specification and misspecification in systems of demand equations: a testing strategy for model selection", Applied Economics, 34, 709-725, 2002. Verbeek M, "A guide to moderm econometrics", 3rd edition, John Wiley, 2008. 


\begin{tabular}{lll}
\hline Antibiotic group & Description of main active ingredients \\
\hline Group 1: & Penicillins (classic) & $\begin{array}{l}\text { Penicillin V, amoxicillin, ampicillin } \\
\text { Group 2: }\end{array}$ \\
$\begin{array}{l}\text { Penicillins (amoxi/clav) and } \\
\text { cephalosporins }\end{array}$ & $\begin{array}{l}\text { Clavucanic acid/amoxicillin, cefaclor, } \\
\text { cefixime, cefadroxil, cefetamet pivoxil, } \\
\text { cefprozil, cefuroxime axetil }\end{array}$ \\
Group 3: $\begin{array}{l}\text { 3rd generation cephalosporins } \\
\text { and quinolones }\end{array}$ & $\begin{array}{l}\text { Ceftibuten, cefpodoxime proxetil, } \\
\text { moxifloxacin, levofloxacin }\end{array}$ \\
Group 4: Macrolides & $\begin{array}{l}\text { Erytromycin, clarithromycin, } \\
\text { roxithromycin, azithromycin }\end{array}$ \\
\hline
\end{tabular}

Table 1: Antibiotic groups used in the treatment of respiratory infections.

\begin{tabular}{ccccc}
\hline & Min & Mean & SD & Max \\
\hline Expenditure share & & & & \\
\hline Group 1 & 0.018 & 0.093 & 0.029 & 0.233 \\
Group 2 & 0.149 & 0.463 & 0.069 & 0.704 \\
Group 3 & 0 & 0.141 & 0.056 & 0.430 \\
Group 4 & 0.154 & 0.303 & 0.073 & 0.787 \\
\hline Price & & & & \\
\hline Group 1 & 0.974 & 1.589 & 0.212 & 2.639 \\
Group 2 & 2.331 & 3.340 & 0.220 & 4.018 \\
Group 3 & 4.974 & 5.935 & 0.291 & 7.451 \\
Group 4 & 3.614 & 4.787 & 0.344 & 6.086 \\
\hline
\end{tabular}

Table 2: Summary statistics of budget shares and prices for the four groups of antibiotics. 


\begin{tabular}{llccc}
\hline Variable & Description & Min & Mean & Max \\
\hline$x / P$ & Income per capita defined in CHF & 15,422 & 23,465 & 51,446 \\
$P O P_{1}$ & Population under 14/total population & 0.088 & 0.166 & 0.218 \\
$P O P_{2}$ & Population between 15 and 25/total population & 0.069 & 0.125 & 0.185 \\
$P O P_{3}$ & Population between 26 and 59/total population & 0.423 & 0.495 & 0.638 \\
$P O P_{4}$ & Population between 60 and 74/total population & 0.095 & 0.136 & 0.249 \\
$P O P_{5}$ & Population over 74/total population & 0.034 & 0.077 & 0.139 \\
$I N F$ & Incidence of common gastrointestinal infections & 91.9 & 114.7 & 187.7 \\
& (Salmonella and Campylobacter) in 100,000 inhab. & & & \\
$I N F 2$ & Incidence of common respiratory infections & 2 & 64.98 & 144 \\
& Streptococcus) in 100,000 inhabitants & & & \\
DLAT & Areas with a Latin culture & - & 0.437 & - \\
\hline$R E S$ & Resistance indicator (pneumococcal susceptibility) & West & Mid & East \\
\hline & Group 1 & 76 & 90.4 & 93.2 \\
& Group 2 & 76 & 100 & 96.1 \\
& Group 3 & 83 & 90.9 & 87.6 \\
& Group 4 & 100 & 97.7 & 69.5 \\
\hline
\end{tabular}

Table 3: Variables notation and summary statistics. 


\begin{tabular}{|c|c|c|c|c|c|c|}
\hline & \multicolumn{2}{|c|}{$\begin{array}{c}\text { Group 2: } \\
\text { Penicillins (amoxi/clav) } \\
\text { and 1st - 2nd generations } \\
\text { cephalosporins }\end{array}$} & \multicolumn{2}{|c|}{$\begin{array}{l}\text { Group 3: } \\
\text { 3rd generation } \\
\text { cephalosporins } \\
\text { and quinolones }\end{array}$} & \multicolumn{2}{|c|}{$\begin{array}{l}\text { Group 4: } \\
\text { Macrolides }\end{array}$} \\
\hline Obs. & \multicolumn{2}{|c|}{720} & \multicolumn{2}{|l|}{720} & \multicolumn{2}{|l|}{720} \\
\hline $\mathrm{R}^{2}$ & \multicolumn{2}{|c|}{0.358} & \multicolumn{2}{|l|}{0.402} & \multicolumn{2}{|l|}{0.479} \\
\hline & Coeff. & S.E. & Coeff. & S.E. & Coeff. & S.E. \\
\hline Constant & $0.2327^{* * *}$ & 0.089 & $0.1514^{* *}$ & 0.068 & 0.0716 & 0.111 \\
\hline$D I D_{-i}$ & $0.6001^{* * * *}$ & 0.035 & $0.6083^{* * * *}$ & 0.040 & $0.5950^{* * * *}$ & 0.036 \\
\hline$P_{1}$ & $0.1502^{* * * *}$ & 0.028 & $-0.0838^{* * * *}$ & 0.021 & $-0.0674^{* * *}$ & 0.021 \\
\hline$P_{2}$ & 0.0009 & 0.011 & $0.0521^{* * * *}$ & 0.010 & $-0.0512^{* * * *}$ & 0.010 \\
\hline$P_{3}$ & $-0.0838^{* * * *}$ & 0.021 & 0.0120 & 0.025 & 0.0126 & 0.019 \\
\hline$P_{4}$ & $-0.0673^{* * *}$ & 0.021 & 0.0126 & 0.019 & $0.1060^{* * * *}$ & 0.025 \\
\hline$x / P$ & 0.0001 & 0.004 & -0.0016 & 0.003 & -0.0041 & 0.004 \\
\hline$P O P_{1}$ & -0.0059 & 0.029 & 0.0076 & 0.023 & 0.0145 & 0.025 \\
\hline $\mathrm{POP}_{2}$ & 0.0343 & 0.027 & 0.0017 & 0.022 & $-0.0395^{*}$ & 0.024 \\
\hline $\mathrm{POP}_{4}$ & 0.0004 & 0.017 & -0.0014 & 0.014 & 0.0221 & 0.015 \\
\hline $\mathrm{POP}_{5}$ & 0.0134 & 0.010 & $0.0159^{* *}$ & 0.008 & $-0.0403^{* * * *}$ & 0.009 \\
\hline$I N F$ & $0.0106^{* *}$ & 0.004 & $-0.0068^{*}$ & 0.004 & -0.0046 & 0.004 \\
\hline$I N F 2$ & -0.0002 & 0.002 & -0.0017 & 0.002 & -0.0021 & 0.002 \\
\hline$D L A T$ & 0.0038 & 0.007 & $0.0121^{* *}$ & 0.005 & $-0.0147^{* *}$ & 0.007 \\
\hline$R E S$ & 0.0004 & 0.000 & $0.0002^{* *}$ & 0.000 & -0.0008 & 0.001 \\
\hline$D T_{2}$ & -0.0011 & 0.005 & 0.0042 & 0.004 & $-0.0145^{* * *}$ & 0.004 \\
\hline$D T_{3}$ & $0.0129^{* *}$ & 0.005 & -0.0020 & 0.004 & $-0.0200^{* * * *}$ & 0.004 \\
\hline
\end{tabular}

Table 4: Parameter estimates for the restricted linear approximate AIDS model of antibiotic groups. 


\begin{tabular}{lccc}
\hline & $\begin{array}{c}\text { Uncompensated } \\
\text { price elasticities }\end{array}$ & $\begin{array}{c}\text { Compensated } \\
\text { price elasticities }\end{array}$ & $\begin{array}{c}\text { Expenditure } \\
\text { elasticities }\end{array}$ \\
\hline $\begin{array}{l}\text { Classic } \\
\text { penicillins }\end{array}$ & -1.005 & -0.903 & 1.050 \\
$\begin{array}{l}\text { Penicillins (amoxi/clav) } \\
\text { and 1st - 2nd generation } \\
\text { cephalosporins }\end{array}$ & -0.683 & -0.209 & 1 \\
$\begin{array}{l}\text { 3rd generation } \\
\text { cephalosporins } \\
\text { and quinolones }\end{array}$ & -1 & & 1 \\
Macrolides & & -0.863 & \\
\hline
\end{tabular}

Table 5: Price elasticities and expenditure elasticities evaluated at the sample mean.

\begin{tabular}{llllc}
\hline & $\begin{array}{l}\text { Classic } \\
\text { penicillins }\end{array}$ & $\begin{array}{l}\text { Penicillins } \\
\text { (amoxi/clav) } \\
\text { and 1st - 2nd gen. } \\
\text { cephalosporins }\end{array}$ & $\begin{array}{l}\text { 3rd generation } \\
\text { cephalosporins } \\
\text { and quinolones }\end{array}$ & Macrolides \\
\hline $\begin{array}{l}\text { Classic } \\
\text { penicillins }\end{array}$ & - & 1 & 4.860 & -0.808 \\
$\begin{array}{l}\text { Penicillins } \\
\text { (amoxi/clav) } \\
\text { and 1st - 2nd gen. } \\
\text { cephalosporins }\end{array}$ & - & - & -0.286 & 0.512 \\
$\begin{array}{l}\text { 3rd generation } \\
\text { cephalosporins } \\
\text { and quinolones }\end{array}$ & - & & & \\
\hline
\end{tabular}

Table 6: Allen elasticity of substitution between two groups of antibiotics. 